\title{
Construccionismo social e investigación. Un binomio indispensable para la creación de innovación en mercadotecnia
}

\section{Social constructionism and research. An indispensable pairing for the creation of innovation in marketing}

DOI: https://doi.org/10.17981/econcuc.41.2.2020.Org.1

Artículo de reflexión.

Fecha de recepción: 09/09/2019

Fecha de devolución: 20/01/2020

Fecha de aceptación: 27/02/2020

Fecha de publicación: 02/03/2020

\section{Ricardo Jimeno Espadas}

Universidad Autónoma Metropolitana.

Xochimilco (México)

rjimeno@correo.xoc.uam.mx

\section{Resumen}

Las personas, sin importar su edad, condición social o actividad económica, están en contacto permanente con los esfuerzos de mercadotecnia. Esto genera una gran cantidad de información subjetiva que puede ser aprovechada para hacer más eficiente las estrategias de mercadotecnia; con ello, se genera un desafío para los procesos de investigación académica y empresarial que buscan incorporar información cualitativa a manera de innovación. Este trabajo tiene como objetivo, argumentar que el construccionismo social es un paradigma adecuado para realizar investigaciones innovadoras en mercadotecnia. La revisión documental y el análisis hermenéutico sirvieron el método. La discusión teórica muestra que, en las definiciones de mercadotecnia, e innovación, se encuentran palabras que son referentes de aspectos subjetivos. Por tanto, la evolución de sus conceptos también es resultado de la incorporación del conocimiento que proviene de otros campos del conocimiento. Concluye que el enfoque del construccionismo social ayuda a comprender la manera en la que, los aspectos sociales, afectan la decisión de compra y el desarrollo de nuevos servicios; impactando de manera positiva en el diseño de estrategias de mercadotecnia.

Palabras clave: Construccionismo social; investigación de mercados; mercadotecnia; innovación en mercadotecnia

\begin{abstract}
People, regardless of age, social status or economic activity, are in constant contact with marketing efforts. This generates a wealth of subjective information that can be leveraged to make marketing strategies more efficient; this creates a challenge for academic and business research processes that seek to incorporate qualitative information in the way of innovation. This work aims to argue that social construction is an appropriate paradigm for conducting innovative research in marketing. Documentary review and hermeneutical analysis served the method. Theoretical discussion shows that, in the definitions of marketing, and innovation, there are words that are referring to subjective aspects. Therefore, the evolution of their concepts is also the result of the incorporation of knowledge that comes from other fields of knowledge. Concludes that the approach to social construction helps to understand how social aspects affect the purchasing decision and development of new services; positive impact on the design of marketing strategies.

Keywords: Social constructionism; market research; marketing; marketing innovation
\end{abstract}




\section{INTRODUCCIÓN}

Partiendo de una visión en la que los individuos y las organizaciones funcionan dentro de un entramado de relaciones, formales e informales, entonces se puede reconocer que permanentemente se establecen enlaces con otros actores; y que estos pueden cambiar dependiendo de las diferentes representaciones sociales que se tenga que asumir.

Ante ello, también hay que considerar que algunos de los roles que desarrollan como persona, coinciden en el espacio físico y mental de otros actores, generando que algunas de estas acciones, estén en correspondencia con diferentes esferas temporales de la vida. También hay que tener en cuenta que, resulta imposible que los seres humanos se libren de las cargas emocionales que cada rol impone; con ello, invariablemente proviene la interiorización de cúmulos de experiencias que afectan el comportamiento humano y sus decisiones.

Estos aspectos resultan fundamentales para la mercadotecnia, y son materia de investigación que se realiza en este campo de conocimiento. Con su ejecución, se identificaron patrones de comportamiento en el proceso de compra, generar registros de información y realizar un análisis empleando diferentes métodos.

Con los resultados, las empresas obtienen valiosa información que desemboca en la incorporación de procesos que pretenden estimular el interés, sea por nuevos productos; por incrementar las ventas de productos presentes en el mercado; o, para conquistar la fidelidad de los individuos hacia los productos, servicios, marcas, e inclusive instituciones.

Sin embargo, el alcance de los resultados obtenidos puede ser mayor; pues se pueden llegar a conocer los motivos que dan origen a las acciones, además del sentido que estas persiguen; y también, los aspectos subjetivos que intervienen en la toma de decisiones. Siendo posible esto cuando la investigación en mercadotecnia incorpora el enfoque del construccionismo social.

Esto representa un hito para el ejercicio de la investigación y para continuar con el desarrollo de la academia, pues figura como una oportunidad para incorporar una posición epistémica diferente a la tradicional, que puede mejorar la compresión y consecuente articulación de las propias actividades de marketing. Los resultados obtenidos con esta perspectiva incluyen aspectos que suelen ser enunciados como subjetivos de la realidad de los individuos y de las organizaciones; ignorar estos aspectos, puede significar el fracaso del esfuerzo de mercadotecnia emprendido por las empresas.

Así es como en este documento, se argumenta que, realizar investigación en mercadotecnia desde una perspectiva del construccionismo social, genera una gran cantidad de información cualitativa, que puede ser aprovechada para mejorar el esfuerzo de mercadotecnia realizado por las empresas. Esto significaría una evolución en la investigación que permite enfrentar los, cada vez más frecuentes cambios en el ambiente que rodean los mercados.

Lo anterior, se traduce en una invitación hacia investigadores y empresas, para que realicen una apertura hacia otros enfoques de investigación. En este caso, el construccionismo social, les puede ayudar a conocer las necesidades, aspiraciones, y 
en general, a comprender el entorno de los individuos que componen el mercado; tras ello, pueden aspirar a obtener un mejor desempeño en las actividades que componen el esfuerzo de mercadotecnia.

\section{DisCUSIÓN}

\section{Evolución de la mercadotecnia}

Tras el paso del tiempo, los conceptos apropiados en la mercadotecnia han tenido una evolución que ha sido orientada hacia la inclusión de mejoras. Quizá el ejemplo más emblemático de ello, es la propuesta de la mezcla de mercadotecnia hecha por Neil Borden en 1953 (American Marketing Association-AMA, 2020). Esquema que hasta la fecha resulta ser el fundamento teórico para la práctica de la mercadotecnia.

Según el manifiesto de la American Marketing Association (AMA, 2020), Borden fue uno de los que, insatisfechos con las mejores prácticas existentes en aquel momento, buscaron respuestas para crecimiento de los negocios e impulso de la mercadotecnia con vista hacia el futuro. Desde entonces, parece que una semilla de adecuación continua, quedo inmersa en el campo de la mercadotecnia.

Así fue como en octubre de 2004, la AMA aprueba una nueva versión de la definición sobre la investigación de mercados; en esta se reconocen varias funciones: 1) Vincular al consumidor, cliente y al público con el vendedor, por medio de información; 2) Identificar y definir oportunidades o problemas de comercialización; 3) Generar, refinar y evaluar las acciones de mercadotecnia; 4) Controlar su desempeño; y 5) Mejorar su comprensión como proceso (AMA, 2017).

Otra muestra del espíritu evolutivo de la mercadotecnia se presentó en julio del 2013, cuando la AMA estableció como definición que la mercadotecnia "es la actividad o grupo de entidades y procedimientos para crear, comunicar, entregar e intercambiar ofertas que tienen valor para los consumidores, clientes socios, y la sociedad en general" (AMA, 2017).

En lo anterior, se pueden apreciar tres cosas: la primera es que, los conceptos implicados en mercadotecnia han evolucionado con el paso del tiempo. La segunda es que, en la definición de Investigación de mercados se reconoce que, es parte de su función mejorar la comprensión. La tercera, es que en la definición de mercadotecnia se contempla el intercambio de valor.

Otro manifiesto proviene de Kotler y Keller (2012), quienes expresan que, sea de "manera formal o informal, las personas y las organizaciones se involucran en un gran número de actividades a las que podemos llamar marketing” (p. 3). En esta expresión, se identifica que las acciones de mercadotecnia tienen gran alcance y que se encuentran inmersas en un complejo entramado social.

El reconocimiento referido en el párrafo anterior, se aprecia mejor cuando los mismos autores revelan que la mercadotecnia "es un proceso social por el cual tantos grupos como individuos obtienen lo que necesitan y desean mediante la creación, oferta y libre intercambio de productos y servicios de valor con otros grupos e individuos" (Kotler \& Keller, 2012, p. 5). 
Ante ello, se considera necesario recordar que, en "lo social" se encuentran diversos actores desempeñando diferentes roles, con distintas necesidades, numerosos conocimientos y diversos aspectos culturales (Latour, 2008; Chumaceiro, Chirinos y Reyes, 2016; Otero y Giraldo, 2017). Asuntos que, vistos desde un enfoque económico tradicional, prácticamente resultan inconmensurables, y quedan ocultas bajo el límite de lo que comúnmente se denomina una transacción comercial.

Entonces, si bien se reconoce que, las actividades de mercadotecnia iniciadas por la empresa se fundamentan en la investigación de mercados; también resulta importante que se contemple que esas actividades no deben ser diseñadas con fin único de incremento de ventas o de obtener un beneficio económico. Pues la empresa debe apostar por la satisfacción y conquista de clientes permanentes, entonces, es cuando los componentes sociales que se encuentran ocultos no pueden ser despreciados.

En un ejemplo sencillo, una empresa que ofrece un servicio de café debe disponer de información suficiente en cantidad y calidad, para poder identificar si los clientes que asisten a una cafetería de franquicia acuden por la necesidad de degustar un café de alta calidad; por necesidades de autoestima o egocentrismo; por la búsqueda de pertenencia social; o simplemente, porque la ubicación del negocio es conveniente.

En el supuesto anterior, existe alta dosis de subjetividad. En un proceso de investigación realizado con un enfoque tradicional, se obtendría la información realizando un levantamiento de encuestas; su interpretación sería cuantitativa, mediante alguna técnica estadística; y, podría ser complementada con algunas mediciones en campo. Posteriormente, en un proceso de integración de escritorio, se adicione el nivel socioeconómico del área estudiada, el flujo de personas que transitan; y finalmente, se redacte el informe final para ser entregado.

Ahora, en el mismo ejercicio de la investigación, dada la información que se pretende obtener, cuyo origen está en la esfera personal del entrevistado, varias de las respuestas podrían ser negadas; y así aceptadas, sin mayor esfuerzo por parte de los ejecutores del estudio. Así que, el resultado sobre la experiencia de visita o consumo del producto podría verse desvirtuada por el método de aproximación y forma de registro de los datos en campo.

Por tanto, incorporar a la empresa información de todo tipo, sea social, cultural, antropológica, económica, geográfica o filosófica; para después ser usada en el diseño, distribución, comercialización del producto o prestación del servicio, se torna relevante. Y es aún más importante, cuando existe la pretensión de que este esfuerzo, sea reconocido por el mercado objetivo; este es el caso de los servicios.

En este punto, resulta importante resaltar que, los servicios se producen con un alto grado de subjetividad, pues su producción y consumo son simultáneos (Kotler, Bowen, Makens, García \& Flores, 2011; Organización de Cooperación y Desarrollo Económicos-OCDE \& Oficina de estadísticas de las Comunidades Europeas-EUROSTAT, 2006; Otero y Giraldo, 2017; Paredes-Chacín, 2017); como ejemplos de estos, se puede mencionar al transporte turístico; los esfuerzos logísticos para brindar seguridad; los servicios prestados en los centros de atención al cliente; inclusive el uso de los servicios sanitarios de un restaurante de lujo. 
Vale decir que, dada la diversidad de deseos; necesidades de los demandantes; complejidad de la producción; razones de consumo simultáneo; y naturaleza subjetiva de las necesidades; se requiere de la existencia de un robusto proceso de conocimiento y comprensión del mercado, acompañado de una adecuada planeación estratégica para que el esfuerzo de mercadotecnia no se disuelva.

\section{Sobre innovación}

Por un lado, se tiene el desarrollo de diferentes conceptos de innovación propuestos por diferentes autores. Cilleruelo (2008), siguiendo un orden de desarrollo temporal, se dio a la tarea de identificar las definiciones del concepto más relevantes; su criterio fue la variedad de palabras consideradas como clave en cada una de las propuestas. Así fue como determinó que seis conceptos eran los más relevantes, entre ellos, el desarrollado por Schumpeter.

$\mathrm{Al}$ respecto, es necesario mencionar que, dada la importancia de la aportación teórica de Schumpeter; esta merece un espacio de análisis independiente. Por lo que no se incluye en este cuadro de definiciones concernientes a la innovación.

TABLA 1.

Autores, año y conceptos de innovación

\begin{tabular}{|c|c|c|}
\hline Autor(es) & Año & Concepto \\
\hline Sherman Gee & 1981 & $\begin{array}{l}\text { Proceso en el cual a partir de una idea, invención o reconocimiento de } \\
\text { necesidad se desarrolla un producto, técnica o servicio útil y es aceptado } \\
\text { comercialmente. }\end{array}$ \\
\hline $\begin{array}{l}\text { Julian Pavón } \\
\text { y Richard } \\
\text { Goodman }\end{array}$ & 1981 & $\begin{array}{l}\text { Conjunto de actividades inscritas en un determinado periodo de tiempo y } \\
\text { lugar que conducen a la introducción con éxito en el mercado, por primera } \\
\text { vez, de una idea en forma de nuevos o mejores productos, servicios o técnicas } \\
\text { de gestión y organización. }\end{array}$ \\
\hline $\begin{array}{l}\text { Richard R. } \\
\text { Nelson y } \\
\text { Sidney G. } \\
\text { Winter }\end{array}$ & 1982 & $\begin{array}{l}\text { Cambio que require un considerable grado de imaginación y constituye una } \\
\text { rotura relativamente profunda con la forma establecida de hacer las cosas y } \\
\text { con ello crea fundamentalmente nueva capacidad. }\end{array}$ \\
\hline $\begin{array}{l}\text { Fernández M. } \\
\text { Machado }\end{array}$ & 1997 & $\begin{array}{l}\text { Es el acto frecuentemente repetido de aplicar cambios técnicos nuevos a } \\
\text { la empresa, para lograr beneficios mayores, crecimientos, sostenibilidad y } \\
\text { competititvidad. }\end{array}$ \\
\hline $\begin{array}{l}\text { Julian Pavón } \\
\text { Morote y } \\
\text { Antonio } \\
\text { Hidalgo } \\
\text { Nuchera }\end{array}$ & 1997 & $\begin{array}{l}\text { El proceso de innovación tecnológica se define como el conjunto de las etapas } \\
\text { técnicas, industriales y comerciales que conducen al lanzamiento con éxito en } \\
\text { el mercado de productos manufacturados, o la utilización comercial de nuevos } \\
\text { procesos técnicos. }\end{array}$ \\
\hline Burt, Perrin & 1995 & $\begin{array}{l}\text { Puede definirse como formas nuevas de hacer las cosas mejor o de manera } \\
\text { diferente, muchas veces por medio de saltos cuánticos, en oposición a } \\
\text { ganacias incrementales. }\end{array}$ \\
\hline $\begin{array}{l}\text { Comisión } \\
\text { Europea }\end{array}$ & 1995 & $\begin{array}{l}\text { Es sinónimo de producir, asimilar y explotar con éxito una novedad, en las } \\
\text { esfereas económica y social, de forma que aporte soluciones inéditas a los } \\
\text { problemas y permita así responder a las necesidades de las personas y de la } \\
\text { sociedad. }\end{array}$ \\
\hline
\end{tabular}

Fuente: Elaboración propia a partir de Cilleruelo (2008). 
Ante esta compilación de definiciones dadas en la tabla 1, es necesario resaltar que, si bien el proceso evolutivo esta ordenado conforme a una escala de tiempo, no significa, necesariamente, que el progreso o adición de elementos tenga correspondencia progresiva y fundada en la definición anterior.

Algo que es posible apreciar, aunque sea de manera, quizá difusa, es que cada una de las definiciones proporcionan una idea; alguna, entre las demás, será más relevante para alguno de los lectores. Sin embargo, hay que valorar que eso está en función de sus intereses, conocimientos y familiaridad que tenga con las palabras que componen la definición; esto es la capacidad de agencia, asunto que será definido más adelante.

Si a la tabla anterior, se le agregan los datos bibliográficos de la publicación donde apareció cada definición, se podrá percibir el sentido que persigue cada propuesta. El sentido se configura con elementos afines, o palabras propias del área de conocimiento dominada cada uno de los autores.

Incluso, el sentido puede dar señales certeras sobre la razón por la cual fue realizado el trabajo; también proporciona información que otorga certeza sobre la correspondencia a un trabajo técnico normativo, académico para formación de recursos humanos, o por ser parte de un proyecto de desarrollo tecnológico, o de creación de teoría, ver Tabla 2.

TABLA 2.

Autores y Título de la publicación donde aparece el concepto

\begin{tabular}{|c|c|}
\hline Autor(es) & Título de la publicación \\
\hline Sherman Gee & $\begin{array}{l}\text { Technology tranfer, Innovatión \& Internacional Competitiveness. } \\
\text { Wiley\&Sons, New York, } 1981 .\end{array}$ \\
\hline $\begin{array}{l}\text { Julian Pavón y Richard } \\
\text { Goodman }\end{array}$ & $\begin{array}{l}\text { Proyecto MODELTEC. La planificación del desarrollo tecnológico, CDTI- } \\
\text { CSIC, Madrid, } 1981 .\end{array}$ \\
\hline $\begin{array}{l}\text { Richard R. Nelson y } \\
\text { Sidney G. Winter }\end{array}$ & $\begin{array}{l}\text { An Evolutionary Theory of Economic Change, Harvard University Press, } \\
\text { Cambridge, 1982. }\end{array}$ \\
\hline Fernández M. Machado & $\begin{array}{l}\text { Gestión tecnológica para un salto en el desarrollo industrial. CDTI-CSIC, } \\
\text { Madrid, } 1997 .\end{array}$ \\
\hline $\begin{array}{l}\text { Julian Pavón Morote y } \\
\text { Antonio Hidalgo Nuchera }\end{array}$ & $\begin{array}{l}\text { Gestión e innovación: Un enfoque estratégico, Ediciones Pirámide, } \\
\text { Madrid, } 1997 .\end{array}$ \\
\hline Burt, Perrin & $\begin{array}{l}\text { Evaluation and future Directions for the job Accommodation Network } \\
\text { (JAN) in Canada. Final Report. Employment Policies and Operations, } \\
\text { HRDC, } 1995 .\end{array}$ \\
\hline Comision & Libro Verde de innovación. \\
\hline
\end{tabular}

Fuente: Elaboración propia a partir de Cilleruelo (2008).

Si la información que proporciona cada una de las tablas se mira de forma excluyente, habrá información que pasa desapercibida, y puede quedar oculta para la interpretación o toma de decisiones; con ello, múltiple información que no se encuentran literalmente escritas, desaparece. La presentación de ambas tablas, así como la disposición de la información que contienen, ejemplifican de manera sencilla, como el 
tratamiento de información limita una interpretación ampliada; y con ello también se limitan las posibilidades de reflexión que puede ser fuente de la innovación.

Y es que la innovación no es el resultado de un proceso que se genera espontáneamente en las organizaciones, ni mucho menos se encuentra presente, como filosofía en todos los directores, estrategas o dueños de la empresa; tampoco se logra con la apropiación de una definición. Por el contrario, es el resultado de una serie de procesos que están, en ocasiones determinados, pero sobre todo de la interacción de actores que intervienen en el proceso. Pero también de las relaciones de conocimiento, experiencia, cultura, etcétera; que son subjetivos y forman parte de la información que posee cada uno de los actores.

Hasta aquí, se han mostrado algunas definiciones propuestas por diferentes autores, esto ha servido para ejemplificar que cuando se presenta la información, aunque ordenada, pero de manera excluyente, se corre el riesgo de realizar una interpretación que, aunque es valiosa, discrimina otra información cualitativa que permite conocer la forma en la que se construyó. Con ello, el sentido que persigue, la capacidad de agencia, y la mayor parte de los aspectos subjetivos que posee la información, se deteriora.

A continuación, se presenta el concepto de innovación instituido por un organismo, y tiene como finalidad, demostrar que, si bien no es de manera acentuada, en el fondo las instituciones si otorgan importancia a los aspectos subjetivos, y les consideran como elementos de mejora y productores de la innovación.

Resulta común que, en la práctica de la administración, y consecuentemente en la mercadotecnia, se tome como eje rector lo descrito en el Manual de Oslo para aquello concerniente a la innovación. Ahí se define que:

Una innovación es la introducción de un nuevo, o significativamente mejorado, producto (bien o servicio), de un proceso, de un nuevo método de comercialización o de un nuevo método organizativo, en las prácticas internas de la empresa, la organización del lugar de trabajo o las relaciones exteriores (OCDE \& EUROSTAT, 2006, p. 56).

Ante el análisis del concepto anterior, se puede llegar a pensar que la innovación es la presentación triunfal y objetiva de una serie de procesos que tuvieron que ocurrir para que esta existiera. Bajo esta misma razón, y para que esto sea posible, es necesario reconocer que existe uno o varios agentes que realizan una serie de actividades, muchas de ellas no determinadas, en diferentes esferas de conocimiento; áreas de gestión; y niveles de organización.

Las actividades innovadoras se corresponden con todas las operaciones científicas, tecnológicas, organizativas, financieras, y comerciales que conducen efectivamente, o tienen por objeto conducir, a la introducción de innovaciones. Algunas... son innovadoras en sí mismas, otras no... pero son necesarias para la introducción de innovaciones. ...incluyen también a las de I+D que no están directamente vinculadas a la introducción de una innovación en particular (OCDE \& EUROSTAT, 2006, p. 57)

Las actividades arriba referidas (científicas, tecnológicas, organizativas, financieras, comerciales), persiguen como fin último la instauración de innovación, pero 
requieren de un espacio de inserción. Así es como OCDE y EUROSTAT (2006), realizan una clasificación de la innovación dependiendo del espacio de aplicación; así se tienen innovaciones de: producto; proceso; organización; y de mercadotecnia; siendo esta última, asunto de este documento.

Si bien el Manual de Oslo deja ver entre líneas que la innovación se genera con la intervención de actores, que, sin duda enfrentan situaciones inéditas, las cuales no se encuentran identificadas en procesos o manuales institucionales. La Comisión Europea señala que las empresas e instituciones deben generar capacidad "para invertir en investigación y desarrollo, educación y formación, información, cooperación, y, de forma más general, en intangibles" (Comisión Europea, 1995, p. 7); entendiendo por intangibles, todos aquellos aspectos subjetivos que la organización pueda ir detectando y que consecuentemente puedan generar beneficio.

Dicho lo anterior, habrá que señalar que los actores realizan acciones necesarias para gestionar y provocar la innovación; muchas de estas actividades tienen un origen subjetivo, no están reconocidas en los procesos, tampoco se encuentran en los manuales de procedimientos y se convierten en hechos generados a partir del entusiasmo y del compromiso del participante, todo para aproximarse a consolidar lo que conciben como innovación.

\section{La visión de Schumpeter, una innovación}

En áreas de conocimiento de la Economía, Teoría administrativa y Teoría de las organizaciones; se reconoce a Schumpeter (1997) por los aportes realizados en estos campos del conocimiento científico (Diamond, 2009; Caro, 2016; Paredes-Chacín, 2017; Pérez, 2019). Aquellos conceptos como "innovación, empresario, 'destrucción creactiva' y la visión pesimista respecto al capitalismo, son aspectos asociados a su nombre" (Galindo, 2008, p. 5).

La visión de Schumpeter ha sido calificada de diversas maneras. Una, entre otras, le refiere como una "forma dinámica de concebir el sistema capitalista que contrata con los modelos de la economía neoclásica tradicional" (Rojas, 2013, p. 3).

Otra es presentada por McCraw (mencionado en Galindo, 2008, p. 5), quien califica su perspectiva como "no lineal" respecto al razonamiento económico; pues su preocupación estaba en "cuestiones de muy diversa índole que iban de aspectos estrictamente económicas, por ejemplo, los ciclos económicos, el desarrollo, etc., hasta el análisis de las ideas económicas de los principales filósofos".

En la visión de Schumpeter, la realidad se compone de una mezcla de ideas que pueden proceder de diferentes campos disciplinares, por tanto, en sus análisis y reflexiones no se realiza una discriminación para quedarse únicamente con aquellos que pertenecen a un área en particular.

Esto permite obtener una visión más amplia de todos los aspectos, incluyendo aquellos que puedan ser calificados como sociales o subjetivos, que intervienen en la construcción de una propuesta de teórica; pues esta debe proporcionar los elementos necesarios para que pueda desembocar en la creación de herramientas que funcionen en varios ámbitos e interconectando varias disciplinas. 
Así, las contribuciones de Schumpeter al desarrollo teórico han despertado el interés de otros investigadores, quienes se apropian de esta visión para buscar sentido a la forma en la que se fue construyendo la teoría administrativa y económica.

Ejemplo de ello, es la apreciación que Galindo (2008) realiza sobre su obra; su explicación incorpora aspectos subjetivos obtenidos tras el análisis de sus escritos, que desde entonces han perfilado la capacidad de agencia que hasta ahora mantienen sus aportaciones.

Galindo (2008) lo expresa así, "resalta el hecho de que en su Historia del Análisis Económico describe a Herbert Spencer como "un tonto" (p. 6). O que la pasión de Adam Smith por la literatura era debida a la ausencia de mujeres" para argumentar sobre el sentido crítico, e hiriente de su pensamiento; que, a pesar de ello, ha llegado a generar una corriente de pensamiento alternante con la tradicional.

Esta perspectiva es construida por "neoschumpeterianos", cuyos participantes han tenido desarrollo en "cuestiones de índole metodológica"; de amplitud teórica incorporando mayor grado de complejidad; "aspectos sociológicos de la innovación y finalmente de la reinassance de Schumpeter" (Galindo, 2008, p. 7).

En Schumpeter, esta forma de percepción, discrepante, pero incluyente a la vez del enfoque tradicional positivista de la administración o economía, dirige su atención "hacia el proceso de crecimiento que se relaciona con la introducción de novedades cualitativas que alteran radicalmente el funcionamiento mismo del sistema" (Rojas, 2013, p. 5).

Según Schumpeter, el simple proceso de incremento o crecimiento acumulativo bien puede ser explicado dentro del marco de la teoría neoclásica tradicional. ... a su parecer no es este tipo de crecimiento ... el que distingue y constituye la verdadera naturaleza del capitalismo. ... el capitalismo es discontinuidad, alteración, novedad, reducción constante de todos los parámetros variables (Rojas, 2013, p. 5).

Ahora, aunque en el párrafo anterior se hace referencia al sistema capitalista, esto resulta ser la menor de las particularidades; pues también resulta ser un ejemplo claro de la capacidad de agencia que posee la oración, y del sentido de la orientación que persigue para ser más incluyente de otros aspectos que producen desenvolvimiento en el propio sistema.

El desenvolvimiento, en nuestro sentido, es un fenómeno característico, totalmente extraño a los que puede ser observado en la corriente circular, o en la tendencia al equilibrio. Es un cambio espontáneo y discontinuo en los cauces de la corriente, alteraciones del equilibrio, que desplazan siempre el estado de equilibrio existente con anterioridad. Nuestra teoría del desenvolvimiento no es sino el estudio de este fenómeno y los procesos que le acompañan (Schumpeter, 1997, p. 75).

Aunque Schumpeter fue principalmente un economista, sus métodos y temas fueron tratados de una manera suficientemente amplia para interesar a otros científicos sociales, repercutiendo en otras áreas del conocimiento, más allá de la economía (Diamond, 2009).

Por tanto, se debe hacer notar que en Schumpeter la ortodoxa a la que comúnmente se hace referencia, solo representa una formalización e interpretación de la tradición 
en el pensamiento económico. Sin embargo, la tendencia con los neoschumpeterianos es que la interpretación teórica se vaya nutriendo, realizándose de una manera moderna y más amplia.

En esta descendencia intelectual, se pueden identificar los postulados de diversos autores, que van desde los realizados por Smith y Ricardo hasta Mill, Marshall y Walras. Además, resulta que la teoría ortodoxa referida, únicamente está relacionada con los métodos de análisis económico tradicionales (Nelson \& Winter, 1982).

Bajo todo lo anterior, parece quedar claro que para generar innovación intervienen múltiples circunstancias; y es que no se puede considerar como el resultado de un proceso establecido en un diagrama de procesos, aunque tampoco se puede dejar todo a la espontaneidad de los actores u organizaciones.

Con su reconocimiento en diagramas, manuales de procesos y otros instrumentos de planeación, apenas se tiene un componente para obtener un mejor desempeño. Pues el correcto rendimiento de la organización, está en función de la intersección de esos procesos declarados con otros aspectos que motivan la innovación, específicamente sobre las capacidades, entendidas en un amplio sentido, que poseen los actores que son parte de la organización y del ambiente en el que se generan estos cruces (Lusthaus, Adrien, Anderson y Carden, 2001; Caro, 2016; Pérez, 2019).

Entonces, si alguna organización pretende generar innovación, además de tener identificados y establecidos sus procesos, también debe acumular, analizar y sintetizar un gran volumen de información interna, o sobre el mercado del cual es participe. Pero además, debe pretender ser parte de los descubrimientos científicos y precursora de la utilización de métodos de investigación que puedan dar cuenta de la forma en la que se generan esas intersecciones (Palomo, 2000; Caro, 2016; Pérez, 2019).

\section{Sobre el construccionismo social}

Ante lo expuesto en los apartados anteriores, el construccionismo social se presenta como un enfoque cualitativo donde se cuestiona todo lo que resulta socialmente aceptado como verdadero o real.

Ante sus ojos, todo "lo que ocurre entre los seres humanos adquiere significado a partir de la interacción social” (Agudelo y Estrada, 2012, p. 364); así los individuos que comparten un contexto y se organizan para desarrollar actividades conjuntas construyen un conocimiento que solo corresponde a su realidad. Rodríguez (2008) aduce que no es cuestión "de construir conocimiento intrapsíquico o interpsíquico, es necesario construir conocimiento por el otro y para el otro, para beneficios de la comunidad y no sólo para beneficios individuales" (p. 84).

En el planteamiento construccionista se consideran cinco supuestos básicos. El primero dice que, "los términos mediante los cuales describimos el mundo y a nosotros mismos no están dictados por los objetos hipotéticos de tales descripciones" (Gergen, 1996, p. 161).

$\mathrm{Y}$ es que, desde el nacimiento el ser humano se intercambia en una serie de conexiones sociales que terminan hasta que se fallece; pero, a pesar de la muerte, muchas de las personas con las que se había entablado alguna relación, aún conservan 
esas conexiones. Toman decisiones, emprenden acciones y transmiten información que están motivadas en sucesos o recuerdos ligados a los recuerdos, sentimientos, sensaciones (Gergen, 2006; Otero y Giraldo, 2017; Chumaceiro, Chirinos y Reyes, 2016); por tanto, el significado de la palabra está siendo afectada por una importante carga de aspectos subjetivos.

Además, con el avance de la tecnología estas conexiones no requieren precisamente de la presencia humana, así que la relación puede construirse a través de objetos abióticos; por tanto, "no existe grupo relevante alguno del que sea posible afirmar que constituye los agregados sociales, ningún componente establecido que pueda usarse como punto de partida incontrovertible" (Latour, 2008, pp. 49-50)2.

El segundo menciona que, "los términos y formas mediante los cuales se obtiene la comprensión del mundo y de las personas mismas son artefactos sociales, productos de intercambios histórica y culturalmente situados entre las personas" (Gergen, 1996, p. 162). Estos términos y formas son transferidos mediante el lenguaje, en cualquiera de sus expresiones, sean documentos, símbolos o testimonios (Latour, 2008; Otero y Giraldo, 2017); cuya transferencia reiterada genera inteligibilidad y dependiendo de la extensión y permanencia temporal, puede generar tradición.

Para el caso de la ciencia, esto genera un problema de congruencia, pues las construcciones teóricas son el resultado de consensos en conclaves de investigadores, cuyas propuestas se acompañan de expresiones de lenguaje. Ahora, se reconoce que estos procesos buscan la objetividad y la transparencia, de ahí la forma en la que se construyen, también pretenden configuraciones de utilidad social, como lo son la capacidad de predicción y el valor de su aplicación.

Sin embargo, no se olvidar que, en sí misma la teoría es el fruto de una construcción social, entonces no puede predecir nada, tampoco aplicar nada, depende de la interpretación de un actor; por tanto, funciona como un instrumento que se manipula. Por consiguiente, la capacidad de predicción está en función de quien la aplica, y lo relevante del resultado también está en función de la satisfacción o enfado que se genera en el intérprete, y no precisamente para la descripción el fenómeno en estudio.

El tercer supuesto estipula que, "La medida en la cual una descripción dada el mundo o de nosotros mismos se mantiene a través del tiempo no depende de la validez empírica de la descripción, sino de las vicisitudes del proceso social" (Gergen, 1996, p. 164). Esto significa que, las formas en la que los actores describen, fuese lo que fuese, pueden ser mantenidas a pesar de que el objeto o situación que origina la descripción haya sufrido cambios; consecuentemente, implica a todos los fenómenos estudiados por la ciencia, y aunque el fenómeno cambie, no necesariamente se introducen cambios o generan repercusión teórica.

Para la investigación que se realiza bajo el enfoque del construccionismo social, este supuesto adquiere vital importancia, pues, resulta común que los resultados de investigaciones sean representados mediante estadísticos y otros instrumentos que

\footnotetext{
2 Bruno Latour desarrolló la Teoría del Actor-Red, misma que deriva en una propuesta metodológica para aproximarse al proceso de construcción de relaciones entre los actores. Ante la puesta de marcha de una investigación bajo el enfoque construccionista, el investigador debe estar atento a lo que él denomina fuentes de incertidumbre. Esta cita representa la primer fuente de incertidumbre "No hay grupos, solo formación de grupos" (Latour, 2008, p. 47).
} 
generan información cuantitativa; cuya pertinencia es resultado de los consensos teóricos previos, dentro de comunidades científicas que se encuentran lejanas o ajenas al fenómeno en estudio. Entonces hay que considerar que dichos consensos, se extienden como una práctica de socialización continua entre sus miembros, generando con ello, la construcción de una realidad, cuya reproducción también tiene una carga de agencia para mantener el prestigio y la veracidad de los actores.

El cuarto establece que, "Como el lenguaje es un subproducto de la interacción, su principal significado se deriva del modo en que está inmerso dentro de patrones de relación" (Gergen, 1996, p. 166). En el construccionismo social, el lenguaje también es considerado como fruto de una construcción que se encuentra cargada de referentes sociales e históricos; su presencia y continua reproducción en situaciones determinadas abona a la construcción de rituales. Ejemplo de ello, es la palabra "amen" que se menciona al final de la oración, o cuando se utiliza como parte de una plegaria en el marco de la religión.

Esto... atrae una atención crítica hacia el modo en que los lenguajes -incluyendo las teorías científicas- son usados dentro de la cultura. ...para el construccionista las muestras de lenguaje son unidades dentro de patrones mayores de relación. ...resultados de modos específicos de vida, de rituales intercambio, de relaciones de control y dominación, etc. (Gergen, 1996, p. 167).

Por tanto, el investigador construccionista debe preguntarse ¿qué función ejerce el enunciado de su verdad en sus relaciones? ¿qué rituales genera? ¿cómo coadyuvan a la construcción de actividades sociales? ¿qué impedimentos produce? ¿qué actores salen perjudicados? ¿quiénes son los beneficiados? entre muchas otras posibles preguntas.

El quinto alude que, "Apreciar las formas existentes de discurso es evaluar patrones de vida cultural; cada evaluación da voz a un enclave cultural dado, y compartir apreciaciones facilita la integración del todo.(Gergen, 1996, p. 167). Esto significa que, las evaluaciones solo son válidas para una comunidad de comprensión determinada, son estos conclaves los que otorgan credibilidad o aceptan los resultados dentro de un marco de relación que mantienen con aquello que se está evaluando.

Pero no hay manera de evaluar certeramente la comprensión en forma ampliada y extendida hacia la cultura de una sociedad. Entonces, el resultado de una investigación, donde se utilizó la teoría económica, solo puede ser validado desde aquellas comunidades del conocimiento que estén relacionadas o superpuestas, como lo pueden ser la administración, las finanzas o la estadística; pero no podría ser desde la medicina, psicología o religión.

A pesar de que los aspectos que identifican estos campos repercuten, al mismo tiempo, en una comunidad de actores que sucesivamente se encuentran en un mismo espacio. Entonces, el construccionismo tiene a cargo, establecer un puente entre diferentes campos que permitan revelar las repercusiones sociales, mismas que, se generan al incorporar diferentes interpretaciones, procedentes de distintos campos disciplinares.

Todo lo anterior, permite mantener una visión ampliada sobre el proceso de investigación, donde se reconocen, el proceso de construcción y los interludios sociales que 
mantienen los actores. Y no únicamente sobre un aspecto en donde se han predestinado limites teóricos y metodológicos.

Vergara (2008), lo expresa de la siguiente manera:

Es de resaltar la importancia que en la actualidad se da desde el construccionismo social a los procesos de intercambio social de narraciones de la realidad definida histórica y culturalmente, y no desde la mirada de construcción individual, y esto es lo que se hace consecuente con la comprensión y estudio de los grupos sociales desde el nodo central y el sistema periférico, pues permite comprender tanto al sujeto como a la sociedad o grupo en el cual está inmerso, dado que el conocimiento es construido a partir de las prácticas socio-culturales en relación con el otro (p. 77).

Consecuentemente, habrá que resaltar que, el construccionismo, a diferencia del enfoque tradicional, pone especial atención en los aspectos que pueden potenciar el cambio; pues si bien las relaciones entre los actores se mantienen, esto es gracias a las expresiones culturales, que pueden cambiar. Hasta este punto, se han expuesto los supuestos que constituyen al construccionismo social como una dimensión epistemológica.

Siendo que, en el construccionismo todo es visto como una construcción, configurada mediante relaciones entre diferentes actores; también se reconoce que se pueden utilizar diferentes alternativas teóricas, que sucesivamente pueden desembocar en construcciones metodológicas. Estas pueden ser modeladas como si fuesen hechas a la medida de una investigación. A continuación, con fines de ejemplificar, se presentan brevemente, algunas propuestas metodológicas acuñadas en el construccionismo social.

Searle (1997) plantea una propuesta para diferenciar los hechos que son parte de la realidad actual; por ello, les divide en brutos, e institucionales. El fundamento es que, los hechos brutos corresponden a la naturaleza y son independientes de los acuerdos y de la influencia cultural propia de las sociedades. Así los hechos brutos se encuentran en la base, sobre la cual se postra una estructura; a través de ella, los hechos institucionales van escalando de acuerdo con el estatus funcional, que es otorgado y representado por concepciones mentales previas; expresiones lingüísticas; y acciones que suelen emprender los actores.

Berger y Luckmann (2003), desarrollan una propuesta en la que el lenguaje y el simbolismo, que utilizan los individuos, se encuentran como nodo inicial para abordar el proceso de investigación. Diferente a lo concebido por John Searle, no realizan ninguna distinción de los hechos; por tanto, el orden social que proponen es el fruto de las interacciones entre los actores, mismas que se van construyendo mediante las acciones que estos van emprendiendo.

La adopción de alguna acción por parte de la sociedad generaría habito, misma que funge como el precedente de la institucionalización; es en ese momento cuando el conocimiento social se vuelve una realidad objetiva, y es trasladado a las siguientes generaciones con ayuda del lenguaje y simbolismo; con ello, permanece en la sociedad como conocimiento real subjetivo.

Goffman (1997), desarrolla un trabajo de investigación utilizando un modelo de analogía teatral con la vida cotidiana. Es una propuesta donde se identifican tres 
papeles primordiales, el primero, corresponde al actor que se desempeña bajo la máscara de un personaje; el segundo, es el conjunto de actores que se desempeñan en torno al papel del primer actor; el tercero, es el público, u otros actores que se encuentran presentes y que no intervienen en el acto.

La puesta en escena se produce cuando los actores se ven obligados a representar papeles sociales y previamente determinados; en ellos, el éxito depende de la cantidad de información que posean sobre los demás actores. Si existiere carencia de información, el actor principal se verá en la necesidad de apoyarse en otros instrumentos que le permitan generar evidencia para reforzar el papel que representa.

Por tanto, con esta perspectiva cualitativa se da por sentado que las verdaderas actitudes, creencias y emociones, se encuentran ocultas en ciertos escenarios; y para descubrirles, deberán ocuparse estrategias que le permitan demostrar expresiones involuntarias, cuya finalidad es impresionar a los demás actores y al público. Tal como se realiza en una obra teatral.

Latour (2008) expone la Teoría del Actor-Red, que se fundamenta en la idea de estar inmersos en un entramado de relaciones, donde los actores, independientemente de su origen biótico o abiótico, son considerados como heterogéneos y poseen capacidad de agencia.

La agencia se define como la capacidad que tienen los objetos para transportar y transmitir información, conservando el sentido que debe guardar una acción, que previamente ha sido otorgada por la sociedad. Idealmente, y sin deformaciones, el sentido es trasladado a través de intermediarios, denominados actantes, cuya función es asumir la reproducción de información guardando sentido original. Esta serie de sucesos subjetivos permiten un orden social, que, con el paso del tiempo queda establecido; es decir, institucionalizado.

La propuesta hace hincapié en que la atención del investigador debe centrarse en las relaciones que establecen los actores; mismas que, ante diferentes situaciones sociales se verán comprometidas. Con ello, también resultan afectados el sentido o capacidad de agencia, comprometiendo la prevalencia, o no, de esas relaciones. Así es como surgen las controversias sociales, mismas que, metodológicamente resultan ser ideales para poder fungir como el punto de inicio de un rastreo de asociaciones.

Lefebvre (2013) desarrolló una teoría nombrada la producción del espacio, en donde argumenta que, el espacio físico en el que se desarrollan las actividades productivas de la sociedad, es socialmente producido. Los orígenes de su planteamiento se encuentran en el Materialismo Dialéctico de Marx y en la Teoría unitaria del espacio; con ello, logra una evolución teórica para desarrollar una crítica sobre el funcionalismo urbano en contextos de consumo dirigido.

En su desarrollo plantea tres dimensiones espaciales, mismas que se encuentran apropiadas por los individuos en diferentes esferas cognitivas. Esta apropiación se genera, cuando los actores establecen enlaces con expresiones simbólicas; conocimientos colectivos; y con las formas de uso del espacio público. Estas dimensiones son: el espacio percibido; el espacio concebido; y el espacio vivido; que sucesiva y respectivamente, están en armonía con la racionalidad física, mental y social del individuo. 
Fridman (2019) desarrolló un trabajo que permite aproximarse al imaginario social, compartido entre comunidades que se encuentran en la búsqueda de la libertad financiera. Utilizando la observación participante, y herramientas de registro propias de la etnometodología, logra descubrir los aspectos que detonan las acciones de emprendimiento que pretenden las personas; esto con independencia de su condición económica y de la actividad a la que financieramente se dedican.

$\mathrm{Su}$ ejercicio de aproximación permite mostrar la forma en la que se van creando redes de colaboración, que funcionan como de escuelas no reconocidas de innovación y emprendimiento, donde aprenden con juegos y ejercicios de simulación sobre escenarios financieros. Con ello, demuestra que el conocimiento social y las consecuentes formas de enfrentar las dificultades financieras, tienen su origen en aspectos subjetivos de aspiración económica y en construcciones sociales.

\section{Sobre innovación teórica en mercadotecnia}

Habrá que recordar, que las estrategias de mercadotecnia no son el resultado exclusivo de procesos administrativos en la organización; y aunque sea común que, en los asuntos de mercadotecnia se involucre el proceso administrativo propuesto por Taylor (1911), las diferencias entre las empresas dependerán de su estructura; formas de organización (Drucker, 1954); y de, la apropiación y desarrollo de conocimiento teórico.

Resulta común que, en la idea de realizar investigación, prevalezca una visión de innovación; con ella, se mantiene que la principal labor de la mercadotecnia es, "satisfacer las necesidades del cliente mediante el producto y todo el conjunto de cosas relacionadas con su creación, entrega y consumo final ... cualquier empresa comienza con los clientes y sus necesidades y no con una patente" (Palomo, 2000, p. 42).

Lo anterior, promueve un reto para la empresa; pues, el cliente también se convierte en un proveedor de información que alimenta el proceso de innovación. Esto proporcionará ventajas a la organización, pues con su incorporación, se "reduce el riesgo de fracaso de un nuevo producto para el mercado" (Palomo, 2000, p. 47).

En esta idea, la empresa fomenta una cultura empresarial, que pone por delante la necesidad del cliente; al mismo tiempo, promueve un proceso de retroalimentación que aporta para la generación de innovación en la empresa.

Así es como, bajo el espíritu de la innovación, se incorporan reflexiones utilizadas en otros campos del conocimiento; inclusive, dentro de la literatura se reconocen algunas tendencias que, quizá en el futuro puedan consolidarse como escuelas. Sin embargo, habrá que reconocer que algunas de estas propuestas resultan ser más arriesgadas, en comparación con otras.

Bajo la corriente que puede tildarse de conservadora, están las propuestas de autores que sostienen que el modelo tradicional de las cuatro "P" (producto, precio, plaza, y promoción), propuesto por McCarthy, es perfectamente adaptable al contexto comercial del internet, y a las tecnologías digitales. Por tanto, es probable que esta visión pueda continuar siendo la dominante en el campo de la mercadotecnia (Dominici, 2009). 
Entre las propuestas que promueven la modificación del esquema tradicional, se encuentran la de Kotler \& Keller (2012), quienes apuestan por un modelo de orientación holística, apoyado en la planeación estratégica. Su principal pretensión es generar una cadena de valor para el cliente, y, en consecuencia, expandir la participación de mercado, generar lealtad, e incrementar el valor de marca.

Con ello, buscan llegar "a un grupo más representativo que abarca las realidades modernas de marketing” (Kotler \& Keller, 2012, p. 25). Para ello, adicionan al modelo tradicional, otras cuatro "P" (personas, procesos, programas, y performance), que estarían plenamente dirigidas hacia la gestión interna de la organización.

Esta adición, se complementa con el desarrollo de estrategias de diferenciación e innovación, que se promueven para mercados determinados. Kotler \& Trías de Bes (2004), proponen que las formas habituales de innovar, están en conformidad con cuatro aspectos; el primero, es la modulación de la estrategia de mercadotecnia; el segundo, la adaptación del tamaño del envase, porción y diseño; el tercero, refiere el desarrollo de complementos; y, el cuarto, pretende la reducción del esfuerzo de mercadotecnia.

Otros autores como Lin \& Chen (2006), se han inclinado por el desarrollo teórico, más que por la modificación del modelo tradicional de la mezcla de mercadotecnia; señalan que existe la necesidad de investigar más sobre la relación existente entre el conocimiento del producto, y la información disponible para el cliente.

Pues, se necesita tener claridad sobre aquellos aspectos que más beneficios reportan a las ventas, y que subyacen en el consumidor. Con ello, se produce un dilema, dificultando la decisión de la empresa para saber si apostar por obtener una gran cantidad de información, o solo recopilar aquella que se considera relevante en el proceso de compra.

Propuestas de mayor riesgo, se configuran con la adición de teorías generadas en otros campos del conocimiento; ejemplo de ello, es la propuesta de Obadă (2013), quien utiliza la Teoría del Flujo, perteneciente al campo de la psicología, para comprender los entornos en línea.

Lo expuesto en este apartado, es una pequeña muestra sobre las inquietudes que existen entre los estudiosos de la mercadotecnia. Sea para, utilizar otros enfoques; incorporar teorías originadas en otros campos; o desarrollar mejores métodos de investigación. Es en este espacio, donde el construccionismo social encuentra un área de oportunidad, y pone a disposición su diversidad (Reyes y Mendoza, 1999); con las cuales, el investigador podría centrar su atención, en las relaciones sociales que se van creando entre las empresas y los clientes.

\section{Conclusiones}

No se puede negar que, en las últimas décadas, los avances tecnológicos han cambiado el entorno de los individuos y de las empresas; estos cambios continuarán. Por lo cual, es necesario que la mercadotecnia, vaya realizando ajustes que permitan dar cuenta de las transformaciones que se van presentando en los mercados, y de sus consecuencias en el consumo. 
El modelo tradicional de las cuatro "P", se conceptualizó en una época en donde el interés del cliente, principalmente, estaba para los productos físicos; y si bien, servicios como el transporte o la atención médica ya existían, era un periodo en donde, ni las empresas, ni los clientes, contaban con la cantidad de información con la que ahora se cuenta. Además, resulta común que este modelo, haya sido utilizado para mantener la gestión interna de la empresa.

En la actualidad, existe suficiente desarrollo de conocimiento teórico para poder centrar la atención de investigadores, en aspectos como, la experiencia vívida; la atención recibida; y en general, en asuntos que, en la antigüedad, incluso eran negados por las organizaciones proveedoras de servicios; pues era común que fuesen tratados como aspectos subjetivos inconmensurables.

A la par, el desarrollo tecnológico ha permitido la proliferación y expansión de ambientes digitales, aspecto que ha derivado en una creciente capacidad de los clientes para interactuar con las organizaciones. Así es como, estos nuevos consumidores se han expresado a favor del desarrollo de productos con extensiones de servicio posteriores a la compra; ante ello, las empresas han buscado estrategias para otorgar seguimiento y soporte a los clientes.

La aparición de propuestas que buscan refrescar la tradicional mezcla de mercadotecnia, comúnmente utiliza la adición de elementos, contextos, y letras, que simbolizan las políticas de gestión; y resultan ser ejemplos de, las diferentes maneras en las que se ha integrado el conocimiento; del reconocimiento de la evolución que han tenido los mercados; del esfuerzo que han realizado las empresas para incorporar las necesidades emergentes.

Es bien sabido que, las organizaciones invierten una parte importante de sus recursos en mejorar procesos, es parte de la forma estratégica para ser eficientes y mejorar su participación en el mercado. Por tanto, es imperante que la ejecución de todo esfuerzo en mercadotecnia sea más eficiente; pues, ante un contexto organizacional, cada vez más complejo, los recursos se tornan cada día más escasos. Si el esfuerzo de mercadotecnia fracasa, la organización entera corre peligro.

Aquí es donde cobra importancia el conocimiento que tenga la organización sobre los factores de decisión de compra, entonces, la investigación que se desarrolla en las empresas ayudaría a entender las nuevas formas de interacción; y a comprender la manera en la que los aspectos sociales afectan la decisión de compra. Por supuesto, también impacta en el desarrollo de nuevos productos, servicios, y en la forma de direccionar las estrategias de mercadotecnia hacia mercados emergentes.

Ante el reto que esto implica, el construccionismo social se presenta como una alternativa, su principal virtud es reconocer la existencia de otros paradigmas, teorías y métodos; además, se torna respetuoso de las diferentes posiciones teóricas que prevalecen en el entorno. Situación que no es posible en los paradigmas tradicionales.

Cuando se utiliza el construccionismo social, se incorporan diferentes posiciones teóricas, y metodológicas de investigación cualitativa; además es posible tomar herramientas generadas en otros campos del conocimiento, lo que permite mejorar la comprensión de información.

Con ello, la empresa puede objetivar información que antes pudo ser considerada como subjetiva, para después difundirla; así los trabajadores, comienzan a habituarse 
y extenderán su participación en una realidad que puede ser considerada como aumentada. Esto funciona como una incubadora de ideas que, mediante una correcta gestión, alimentan el proceso de innovación.

Lo anterior es posible, si se considera que la vida cotidiana de los actores, está compuesta con versiones que provienen de diferentes campos teóricos; entonces, al generar discursos que resultan de interés transversal, se mejora la comprensión de los puntos de vista de aquellos que intervienen. Así, no solo los aspectos antropológicos y sociales se perciben útiles en la sociedad, sino que también, enriquecen la toma decisiones en conformidad con los procesos comerciales.

Es importante acotar, que las acciones de mercadotecnia se encuentran presentes en todo momento dentro del entramado social. Entonces, con la adecuada lubricación teórica que brinda el construccionismo, la mercadotecnia puede funcionar como un caleidoscopio; cuyo conocimiento toca al mismo tiempo, muchos nodos que componen nuestro contexto.

Con ello, el investigador, y sucesivamente la empresa, se vuelven capaces de utilizar cualquier tipo de información, adecuando planteamientos sin desestimar aspectos que resultan importantes para diferentes grupos sociales. Si esto se traslada al mercado, se puede ser más eficientes en la aplicación del esfuerzo de mercadotecnia.

\section{REFERENCIAS}

Agudelo, M. E. y Estrada, P. (2012). Constructivismo y construccionismo social: Algunos puntos comunes y algunas divergencias de estas corrientes teóricas. Prospectiva, (17), 353-378. https://doi.org/10.25100/prts.v0i17.1156

AMA. (2017). Definition of Marketing. Chicago: American Marketing Association. [Online]. https://www.ama.org/AboutAMA/Pages/Definition-of-Marketing.aspx

AMA. (2020). About AMA. Chicago: American Marketing Association. [Online]. Disponible en https://www.ama.org/about-ama/

Berger, P. y Luckmann, T. (2003). La construcción social de la realidad. (18 $E d$.). Buenos Aires: Amorrortu.

Caro, J. (2016). Financiación de la innovación tecnológica en el sector servicios en Colombia. Económicas CUC, 37(2), 89-114. https://doi.org/10.17981/econcuc.37.2.2016.05

Chumaceiro, A., Chirinos, E. \& Reyes, I. (2016). History of political and social organizations aimed at promoting corporate social responsibility in the twentieth century. Búsqueda, 3(16), 20-32. https://doi.org/10.21892/01239813.163

Cilleruelo, E. (2008). Compendio de definiciones del concepto $<<$ Innovación $>>$ realizadas por autores relevantes: Diseño híbrido actualizado del concepto. Dirección y Organización, Octubre(36), 61-68. Disponible en https://revistadyo.es/DyO/index. php/dyo/article/view/71

Comisión Europea. (1995). Libro verde de la Innovación. Luxemburgo: Comision Europea. Recuperado de https://sid.usal.es/idocs/F8/FDO11925/libroverde.pdf

Diamond, A. M. (2009). Shumpeter vs Keynes: In the long run not all of are dead. Journal of the History of Economic Thought, 31(4), 531-541. https://doi.org/10.1017/ S1053837209990307 
Dominici, G. (2009). From Marketing mix to E-Marketing mix: a literature overview and classification. International Journal of Business and Managment, 4(9), 17-24. https://doi.org/10.5539/ijbm.v4n9p17

Drucker, P. F. (1954). The practice of management. New York: Harper \& Row.

Fridman, D. (2019). El sueño de vivir sin trabajar Una sociología del emprendedorismo, la autoayuda financiera y el nuevo individuo del siglo XXI. Tres Cantos: Siglo XXI Editores.

Galindo, M. Á. (2008). Schumpeter: Vida y pensamiento de un polemista. Libros de Economía y Empresa, 3(7), 5-7. Recuperado de http://www.librosdeeconomiayempresa.com/r007/RevistaNumero7CajaDueroLibrosEconomia.pdf

Gergen, K. J. (2006). El yo saturado Dilemas de identidad en el mundo contemporáneo. Barcelona: Paidós.

Gergen, K. J. (1996). La construcción social: emergencia y potencial. En, M. Pakman, Construcciones de la experiencia humana. Barcelona: Gedisa.

Goffman, E. (1997). La presentación de la persona en la vida cotidiana. (3a ed.). Buenos Aires: Amorrortu.

Kotler, P., Bowen, J. T., Makens, J. C., García, J. \& Flores, J. (2011). Marketing turístico. (5a ed.). London: Pearson Educación.

Kotler, P. y Keller, K. L. (2012). Dirección de Marketing. (14 a ed). Londres: Pearson Educación.

Kotler, P. \& Trías, F. (2004). Marketing lateral. London: Pearson Educación.

Latour, B. (2008). Reensamblar lo social. Una introducción a la teoría del actor-red. Buenos Aires: Manantial.

Lefebvre, H. (2013). La producción del espacio. Madrid: Capitán Swing Libros.

Lin, L. \& Chen, C.-S. (2006). The influence of the country-of-origin image, product knowledge and product involvement on consumer purchase decisions: an empirical study of insurance and catering services in Taiwan. Journal of Consumer Marketing, 23(5), 248-265. https://doi.org/10.1108/07363760610681655

Lusthaus, C., Adrien, M.-H., Anderson, G. y Carden, F. (2001). Mejorando el desempeño de las organizaciones Método de autoevaluación. [En linea]. Recuperado de http://www.bio-nica.info/Biblioteca/Lusthaus2001MetodoAutoevaluacion.pdf

Nelson, R. R. \& Winter, S. G. (1982). An evolutionary theory of economic change. Boston: Harvard University Press. Recuperado de http://inctpped.ie.ufrj.br/spiderweb/pdf_2/Dosi_1_An_evolutionary-theory-of_economic_change..pdf

Obadă, D. (2013). Flow theory and online marketing outcomes: a critical literature review. International Economic Conference of Sibiu, post Crisis Economy: Challenges and Opportunities, 6, 550-561. https://doi.org/10.1016/S22125671(13)00173-1

OCDE \& EUROSTAT. (2006). Manual de Oslo Guia para la recogida e interpretación de datos sobre innovación. (3a ed.). Madrid: Grupo Tragsa \& Empresa de Transformación Agraría S.A.

Otero, M. y Giraldo, W. (2017). Consumo de productos infantiles en la base de la pirámide poblacional: análisis de los mecanismos influyentes. Económicas CUC, 38(1), 165-184. https://doi.org/10.17981/econcuc.38.1.08 
Palomo, M. A. (2000). Consumo de productos infantiles en la base de la pirámide poblacional: análisis de los mecanismos influyentes. Ingenierías, 3(8), 41-48. Recuperado de http://www.ingenierias.uanl.mx/8/pdf/8_Miguel_Palomo_El_proceso_marketing.pdf

Paredes-Chacín, J. (2017). Planificación financiera ante la perspectiva organizacional en empresas cementeras del estado Zulia-Venezuela. Económicas CUC, 38(1), 105132. https://doi.org/10.17981/econcuc.38.1.05

Pérez, C. (2019). Innovación empresarial al servicio de la micro y pequeña empresa nortesantandereana: por la competitividad regional. Económicas CUC, 40(1), 91104. https://doi.org/10.17981/econcuc.40.1.2019.06

Reyes, R. y Mendoza, R. (1999). De la ontología muda a las retóricas de la calidad: aproximaciones al construccionismo social. Administración y Organizaciones, 3(1), 69-86. Disponible en https://rayo.xoc.uam.mx/index.php/Rayo/issue/archive

Rodríguez, H. (2008). Del constructivismo al construccionismo: implicaciones educativas. Revista Educación y Desarrollo Social, 2(1), 71-89. Disponible en https:// revistas.unimilitar.edu.co/index.php/reds/article/view/710

Rojas, M. (2013). Recordando a Joseph Schumpeter. Una introducción a la obra de Schumpeter a 130 años de su nacimiento. Ensayos de la biblioteca Virtual de Mauricio Rojas, (15). Recuperado de https://bibliotecademauriciorojas.files.wordpress.com/2012/04/m-rojas-recordando-a-joseph-schumpeter.pdf

Schumpeter, J. A. (1997). Teoría del desenvolvimiento económico Una investigación sobre ganancias, capital, crédito, interés y ciclo económico. (2a ed.). México, D.F.: FCE.

Searle, J. R. (1997). La construcción de la realidad social. Barcelona: Paidos.

Taylor, F. (1911). The principles of scientific management. New York: Cosimoclassics.

Vergara, M. (2008). La naturaleza de las representaciones sociales. Revista latinoamericana de ciencias sociales, niñez y juventud, 6(1), 55-80. Recuperado de http:// biblioteca.clacso.edu.ar/Colombia/alianza-cinde-umz/20130801104940/ArtMariadelCarmenVergara.pdf

\section{BIODATA}

Ricardo Jimeno Espadas es Doctor en Estudios Organizacionales por la Universidad Autónoma Metropolitana (Ciudad de México, México). Doctor en Administración de la Universidad EAFIT (Medellín, Colombia). Maestro en Mercadotecnia Turística por la Universidad la Salle (Cancún, México). Licenciado en Administración del Instituto Tecnológico de Cancún (México). Profesor-Investigador adscrito a la Universidad Autónoma Metropolitana, Unidad Xochimilco, en el Departamento de Producción Económica. (Ciudad de México, México). https://orcid.org/0000-0003-3625-729X 